\title{
A Generalized Bilateral Trading Model
}

\author{
Rongchuan Tao \\ School of Economy and Management, Wuhan University, Wuhan, China \\ Email: RongchuanTao@whu.edu.cn
}

How to cite this paper: Tao, R.C. (2018) A Generalized Bilateral Trading Model. Open Journal of Business and Management, 6, 606-615.

https://doi.org/10.4236/ojbm.2018.63046

Received: May 20, 2018

Accepted: June 9, 2018

Published: June 12, 2018

Copyright $\odot 2018$ by author and Scientific Research Publishing Inc. This work is licensed under the Creative Commons Attribution International License (CC BY 4.0).

http://creativecommons.org/licenses/by/4.0/

\begin{abstract}
In the bilateral trading model developed by Myerson [1], an ex post efficient mechanism that satisfies incentive compatibility and individual rationality has been proved to be not existed. This paper is aimed at the discussion of modified bilateral trading model where the buyer and seller's valuations for the object are assumed to be not independent. Under such assumption, we concern about the existence of an ex post efficient mechanism satisfying individual compatible and individual rational conditions. We will give a necessary condition of such mechanism, not precisely though, to identify the existence. At the end of this paper, we provide some empirical evidences about the non-independent assumption and suggest some directions for further research, both empirical and theoretical.
\end{abstract}

\section{Keywords}

Bilateral Trading, Mechanism Design, Individual Rationality, Incentive Compatibility, Ex Post Efficient, Generalized Model

\section{Introduction}

Uncertainty and asymmetric information have been under central discussion in mechanism design and auction theories. Many theories have been put forward to model the behavior of market or mechanism under asymmetric information, which can be traced back to the research in Lemon market and adverse selection of job market [2] [3]. One classic model among asymmetric information models is principal-agent model [4]. Bilateral trading model can be treated as a special case of principal-agent model, and can be analyzed by the frame of it. One of the most important ideas of principal-agent model is to indicate that under asymmetric information, principal should pay extra money in order to make every agent report his type honestly and willing to participate in the game. The extra cost is evaluated as information rent. But cases are not totally the same in bilateral trading model. The difference lies in that there is no principal, and we only 
need to design a mechanism or a tariff to ensure each player is willing to participate in and the mechanism exhibits desirable features in maximizing the net utility of both players. Such normal mechanism is characterized in terms of individual rationality, incentive compatibility, and ex post efficiency. We shall explain them later.

In Vickrey-Clarke-Groves (VCG) auction mechanism design, the optimal auction mechanism is clear for a class of distribution where agents types are independently and identically distributed, which has been proved in references [5] and [6]. Independent distribution serves a central condition for the computation of agents' virtual payoff.

Previous research about bilateral trading problem ([7] [8]) or optimal auction design showed the impossibility of designing a mechanism that is individual rational, incentive compatible and ex post efficient without outside subsidy. Individual rationality, incentive compatibility, and ex post efficiency are important criterion for a well designed mechanism. If a mechanism is not individual rational, the market will crash since players cannot receive positive expected utility. If a mechanism is not incentive compatible, the players won't honestly report their real type. If a mechanism is not ex post efficient, there would be some situations where trade determined by the mechanism such that each player must attain negative utility. The key element that causes such outcome is the assumption about the joint distribution of the participator's valuation, that is: the valuation random variables $v_{1}$ and $v_{2}$ are independent. The assumption directly implies that the trading area is a rectangle $\left[a_{1}, b_{1}\right] \times\left[a_{2}, b_{2}\right]$. The proof of the negative expected utility of the point $\left(b_{1}, a_{2}\right)$ indicates that even a mechanism satisfies the incentive compatible and ex post efficient, it would fail to meet each player's individual rationality. So the market can only support an individual rational, incentive compatible and ex post efficient mechanism with strictly positive subsidy.

We can generalize the model by relaxing this assumption. Now the random variables $V_{1}$ and $V_{2}$ are distributed on a convex area $D$. So if we can prove that each point $(x, y)$ in area $D$ yields positive utility for each player, i.e.

$U_{1}(x)+U_{2}(y)>0$, we can prove the existence of the corresponding mechanism. This paper gives theoretical analyses about the generalized trading model, identifying the existence of such a mechanism.

This paper is organized in the following pattern: in Section 2, we present the basic definition of the modified model, define some basic parameters for further analysis. Part 3 repeated the analysis that is common for a principal-agent model under the changing in distribution. The expressions of incentive compatible and individual rational are slightly different. In Section 4, we argued our main result for generalized bilateral trading model: there may exist an individual rational, incentive compatible and ex post efficient mechanism when the distribution of the type of each player is not independent anymore. Furthermore, in Section 5, we explained the reasons that guarantee the possibility of the existence of such generalized case in an empirical way. The conclusion is shown in part 6. 


\section{Basic Settings of Generalized Model}

Consider a trading problem where individual $\mathbf{1}$ is a buyer and individual $\mathbf{2}$ is a seller. Seller owns an object that buyer wants. Each individual has his own valuation about the object.

Denote the seller's valuation of the object: $V_{1}$, the buyer's valuation of the object: $V_{2} . V_{1}, v_{2}$ are random variables distributed on a convex differentiable zone $D$ with density function $f\left(v_{1}, v_{2}\right)$. The utility of the seller is $v_{2}-X$ and the utility of the buyer is $X-V_{1}$ if the mechanism specifies $x$ as the executing price.

According to an essential result of bargain game called revelation principle, a mechanism can be determined by two functions: $x\left(v_{1}, v_{2}\right), p\left(v_{1}, v_{2}\right)$ if the mechanism is a direct bargaining mechanism.

$x\left(v_{1}, v_{2}\right):$ the price of the object if buyer has valuation $v_{1}$ and the seller has valuation $v_{2}$.

$p\left(v_{1}, v_{2}\right)$ : the possibility of the occurrence of trade if buyer and seller's valuation are $v_{1}$ and $v_{2}$ respectively.

The difference lies in the definition domain of $x\left(v_{1}, v_{2}\right), \quad p\left(v_{1}, v_{2}\right)$ is now on the area $D$.

Given any equilibrium of any bargain game, there exist an equivalent incentive-compatible direct mechanism by first asking the buyer and seller each to confidentially report his valuation, then determine the corresponding $x\left(v_{1}, v_{2}\right)$, $p\left(v_{1}, v_{2}\right)$ by computing according to original mechanism. When we are analyzing bilateral trading mechanism, we only consider such direct mechanism.

We need to define some new variables in order to get the expected utility of buyer and seller. But before giving the specific definition, we need to do some technical work.

Suppose the smallest rectangle $R$ containing $D$ is $\left[a_{1}, b_{1}\right] \times\left[a_{2}, b_{2}\right]$. The following figure shows an example of such a rectangle. Then we extend the definition domain of $f\left(v_{1}, v_{2}\right), p\left(v_{1}, v_{2}\right)$ and $x\left(v_{1}, v_{2}\right)$ to $\left[a_{1}, b_{1}\right] \times\left[a_{2}, b_{2}\right]$. The specific method is: If $\left(v_{1}, v_{2}\right) \in D$, then $f, p, x$ take original value. If $\left(v_{1}, v_{2}\right) \notin D$, then $f, p, x$ equal to 0 . We use the extended $f, p, x$ in the following discussion.

As is shown in Figure 1 , the smallest rectangle $R$ containing $D$ is $\left[a_{1}, b_{1}\right] \times\left[a_{2}, b_{2}\right]$.

We denote the red curve as $L$, which is a part of $D$ 's boundary and is tangent to line: $y=a_{2}$ and line: $x=b_{1}$.

After the above technical works, the buyer and seller's conditional expectation of $x, p$ and their final utility can be written as:

$$
\begin{gathered}
\bar{x}_{1}\left(v_{1}\right)=\frac{\int_{a_{2}}^{b_{2}} x\left(v_{1}, t_{2}\right) f\left(v_{1}, t_{2}\right) \mathrm{d} t_{2}}{h_{1}\left(v_{1}\right)}, \bar{x}_{2}\left(v_{2}\right)=\frac{\int_{a_{1}}^{b_{1}} x\left(t_{1}, v_{2}\right) f\left(t_{1}, v_{2}\right) \mathrm{d} t_{1}}{h_{2}\left(v_{2}\right)}, \\
\bar{p}_{1}\left(v_{1}\right)=\frac{\int_{a_{2}}^{b_{2}} p\left(v_{1}, t_{2}\right) f\left(v_{1}, t_{2}\right) \mathrm{d} t_{2}}{h_{1}\left(v_{1}\right)}, \bar{p}_{2}\left(v_{2}\right)=\frac{\int_{a_{1}}^{b_{1}} p\left(t_{1}, v_{2}\right) f\left(t_{1}, v_{2}\right) \mathrm{d} t_{1}}{h_{2}\left(v_{2}\right)}, \\
U_{1}\left(v_{1}\right)=\bar{x}_{1}\left(v_{1}\right)-v_{1} \bar{p}_{1}\left(v_{1}\right), U_{2}\left(v_{2}\right)=v_{2} \bar{p}_{2}\left(v_{2}\right)-\bar{x}_{2}\left(v_{2}\right),
\end{gathered}
$$




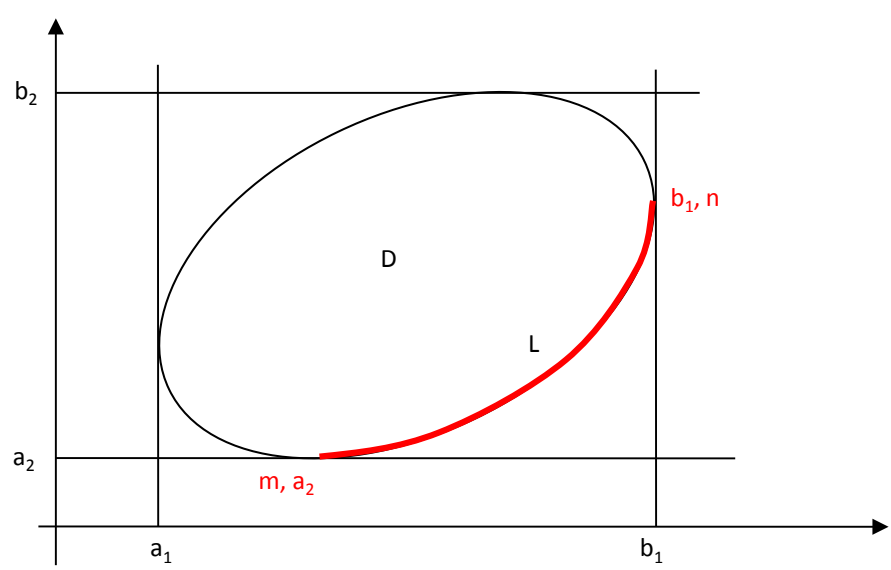

Figure 1. The distribution area $\mathbf{D}$ in the coordinate $\mathrm{v}_{1}-\mathrm{v}_{2}$.

$$
h_{1}\left(v_{1}\right)=\int_{a_{2}}^{b_{2}} f\left(v_{1}, t\right) \mathrm{d} t, h_{2}\left(v_{2}\right)=\int_{a_{1}}^{b_{1}} f\left(t, v_{2}\right) \mathrm{d} t .
$$

The $U_{1}, U_{2}$ defined above is the expected total utility gain from the trade. Thus their economic meanings are well defined. We may call $h_{1}\left(v_{1}\right), h_{2}\left(v_{2}\right)$ : the generalized density function of each individual.

\section{Individual Rationality and Incentive Compatibility}

We first review the definition of incentive compatibility and individual rationality.

\section{Incentive compatibility:}

A mechanism $(p, x)$ is individual compatible iff for any $v_{1}$ and $v_{1}^{\prime}$ in $\left[a_{1}, b_{1}\right]$

$$
U_{1}\left(v_{1}\right) \geq \bar{x}_{1}\left(v_{1}^{\prime}\right)-v_{1} \bar{p}_{1}\left(v_{1}^{\prime}\right)
$$

And for any $v_{2}$ and $v_{2}^{\prime}$ in $\left[a_{2}, b_{2}\right]$

$$
U_{2}\left(v_{2}\right) \geq v_{2} \bar{p}_{2}\left(v_{2}^{\prime}\right)-\bar{x}_{2}\left(v_{2}^{\prime}\right)
$$

The economic interpretation of incentive compatibility is that each of the players can maximize their expected utility by reporting their type honestly, rather than telling a lie.

We can derive that any incentive compatible mechanism must satisfy:

$$
U_{1}\left(v_{1}\right)=U_{1}\left(b_{1}\right)+\int_{v_{1}}^{b_{1}} \bar{p}_{1}\left(t_{1}\right) \mathrm{d} t_{1}, U_{2}\left(v_{2}\right)=U_{1}\left(a_{2}\right)+\int_{a_{2}}^{v_{2}} \bar{p}_{2}\left(t_{2}\right) \mathrm{d} t_{2}
$$

The above relations are natural expansion of the classic results developed in optimal auction design and VCG mechanism design [1].

\section{Individual rationality:}

Also known as participating constraint, Individual rationality requires that, in a game, each player has positive expected utility:

$U_{1}(x)+U_{2}(y) \geq 0$ for any $(x, y)$ in $D$.

If a mechanism is individual rational, players will obtain non-negative expected utility by participating the game.

Here is the main result of the generalized model: 


\section{Theorem:}

For any incentive compatible mechanism in generalized bilateral trading model. The probability function $p\left(v_{1}, v_{2}\right)$ satisfies $\bar{p}_{1}\left(v_{1}\right)$ is decreasing on $v_{1}$, $\bar{p}_{2}\left(v_{2}\right)$ is increasing on $v_{2}$. Moreover, the mechanism satisfies individual rationality condition iff for any point $(x, y)$ on the boundary of $D$. $U_{1}(x)+U_{2}(y) \geq 0$. At last, for any point $(x, y)$ on $L$, we have:

$$
\begin{aligned}
& U_{1}(x)+U_{2}(y) \\
& =\iint_{D}\left(v_{2}-v_{1}\right) f\left(v_{1}, v_{2}\right) p\left(v_{1}, v_{2}\right) \mathrm{d} v_{1} \mathrm{~d} v_{2}-\int_{a_{1}}^{b_{1}} H_{1}\left(t_{1}\right) \bar{p}_{1}\left(t_{1}\right) \mathrm{d} t_{1} \\
& -\int_{a_{2}}^{b_{2}}\left(1-H_{2}\left(t_{2}\right)\right) \bar{p}_{2}\left(t_{2}\right) \mathrm{d} t_{2}+E_{1}+E_{2}
\end{aligned}
$$

And we have the definition of $H_{1}, H_{2}, E_{1}, E_{2}$ :

$$
\begin{gathered}
H_{1}(t)=\int_{a_{1}}^{t} h_{1}(x) \mathrm{d} x, \quad H_{2}(t)=\int_{a_{2}}^{t} h_{2}(x) \mathrm{d} x \\
E_{1}=\int_{x}^{b_{1}} \bar{p}_{1}\left(t_{1}\right) \mathrm{d} t_{1}, \quad E_{2}=\int_{a_{2}}^{y} \bar{p}_{2}\left(t_{2}\right) \mathrm{d} t_{2}
\end{gathered}
$$

A simple deduction of the theorem is: if a mechanism is individual rational, then for any point $(x, y)$ on the line $L$ :

$$
\begin{aligned}
& U_{1}(x)+U_{2}(y) \\
& =\iint_{D}\left(v_{2}-v_{1}\right) f\left(v_{1}, v_{2}\right) p\left(v_{1}, v_{2}\right) \mathrm{d} v_{1} \mathrm{~d} v_{2}-\int_{a_{1}}^{b_{1}} H_{1}\left(t_{1}\right) \bar{p}_{1}\left(t_{1}\right) \mathrm{d} t_{1} \\
& -\int_{a_{2}}^{b_{2}}\left(1-H_{2}\left(t_{2}\right)\right) \bar{p}_{2}\left(t_{2}\right) \mathrm{d} t_{2}+E_{1}+E_{2} \geq 0
\end{aligned}
$$

Due to the monotonic proposition of $\bar{p}_{1}\left(v_{1}\right), \bar{p}_{2}\left(v_{2}\right)$, for any point $(m, n)$ in area $D$, there exist a point $(x, y)$ on $L$ s.t.

$U_{1}(x)+U_{2}(y) \geq U_{1}(m)+U_{2}(n)$. Thus the deduction holds.

Tips: The proof of theorem 1 is again similar to the proof of Myerson [1] Theorem 1. So we don't present the detailed proof of theorem 1 here.

\section{The Existence of an Ex Post Efficient Mechanism}

Indeed, an incentive compatible and individual rational mechanism is desirable for practical usage. But in order to consider the efficiency of a mechanism, we need to consider one more property, which is ex post efficiency. In general, even if a mechanism is incentive compatible and individual rational, the inefficient case where the valuation $v_{1}>v_{2}$ but the mechanism still tells that a trade should be made may appear, which will cause each player becoming worse off.

First, we define another concept that may help us to measure the efficiency of a mechanism.

A mechanism is ex post efficient if and only if

$$
p\left(v_{1}, v_{2}\right)= \begin{cases}1 & \text { if } v_{1} \leq v_{2} \\ 0 & \text { if } v_{1}>v_{2}\end{cases}
$$

It has been proved that for the independent distributed valuation model de- 
veloped in Myerson [1], if a mechanism is incentive compatible and ex post efficient. It must fail to satisfy individual rationality. Now we reconsider this problem in generalized model.

The main difficulty of making a quick justification of the existence of an incentive compatible, individual rational, and ex post efficient mechanism because we need to make sure that any point $(x, y)$ on $L$, satisfies $U_{1}(x)+U_{2}(y) \geq 0$. So we need to find the set of $(x, y)$ that minimize $U_{1}(x)+U_{2}(y)$.

A special subarea of $D$ raised our attention. Suppose line: $y=x$ intersect the boundary of area $D$ at $\left(x_{1}, x_{1}\right),\left(x_{2}, x_{2}\right)$ and without loss of generality, we suppose $x_{1}<x_{2}$. The special area if $D$ is the intersection of $D$ and domain $\left[x_{2}, b_{1}\right] \times\left[a_{2}, x_{1}\right]$, denoting $S$.

$\iint_{D}\left(v_{2}-v_{1}\right) f\left(v_{1}, v_{2}\right) p\left(v_{1}, v_{2}\right) \mathrm{d} v_{1} \mathrm{~d} v_{2}-\int_{a_{1}}^{b_{1}} H_{1}\left(t_{1}\right) \bar{p}_{1}\left(t_{1}\right) \mathrm{d} t_{1} \quad$ is always negative, which contradicts individual rational condition (Figure 2 ).

If $S$ is not empty set, then there is no incentive compatible, individual rational, and ex post efficient mechanism simply because $E_{1}+E_{2}$ is always zero. In fact, we can assure that:

$\iint_{D}\left(v_{2}-v_{1}\right) f\left(v_{1}, v_{2}\right) p\left(v_{1}, v_{2}\right) \mathrm{d} v_{1} \mathrm{~d} v_{2}-\int_{a_{1}}^{b_{1}} H_{1}\left(t_{1}\right) \bar{p}_{1}\left(t_{1}\right) \mathrm{d} t_{1} \quad$ is always negative, which contradicts individual rational condition.

We have identified a necessary condition for the existence of an incentive compatible, individual rational, and ex post efficient mechanism, but we also need some more accurate method to estimate the value of $U_{1}(x)+U_{2}(y)$.

Take derivation of $U_{1}(x)+U_{2}(y)$ with respect to $x$ and $y$. we can get the following equation. This relies on the assumption that $D$ is a differentiable area.

We consider the first order condition:

$$
-\bar{p}_{1}(x) \mathrm{d} x+\bar{p}_{2}(y) \mathrm{d} y=0
$$

which can be also written as:

$$
\frac{\bar{p}_{1}(x)}{\bar{p}_{2}(y)}=\frac{\mathrm{d} y}{\mathrm{~d} x}
$$

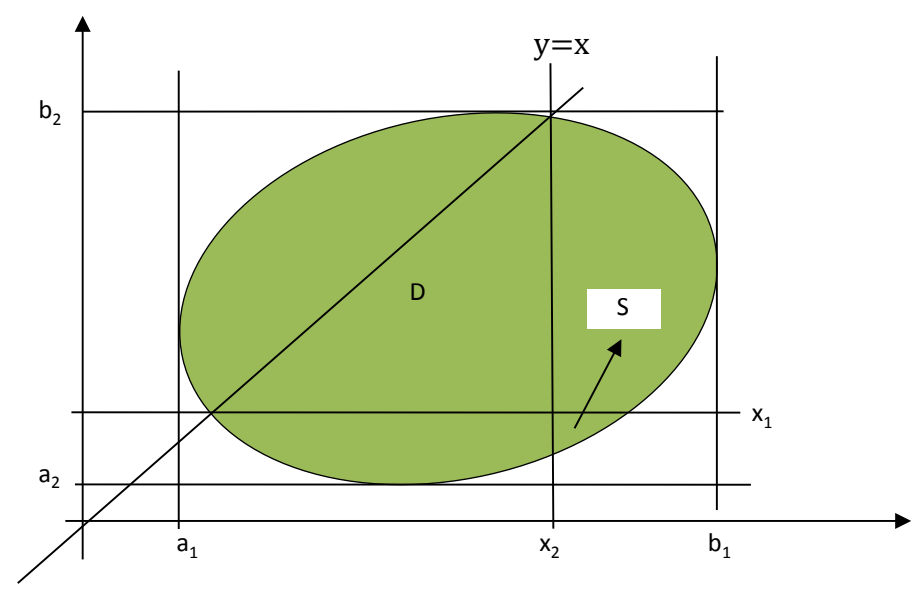

Figure 2. The case where $S$ is not empty set. 
Next we will prove that the first order condition has one and only one solution:

Notice that $\bar{p}_{1}(x)$ is decreasing on $x$, while $\bar{p}_{2}(y)$ is increasing on $y$, and the shape of $L$ ensures that $y$ is increasing on $x$, thus $\frac{\bar{p}_{1}(x)}{\bar{p}_{2}(y)}$ is decreasing on $x$.

Also notice that $\frac{\mathrm{d} y}{\mathrm{~d} x}$ is increasing on $x$ since $D$ is convex. So $\frac{\bar{p}_{1}(x)}{\bar{p}_{2}(y)}-\frac{\mathrm{d} y}{\mathrm{~d} x}$ must have only one zero point and the solution is a minimum value point. So, given a distribution of $V_{1}$ and $V_{2}$, we can numerically calculate the minimum $U_{1}(x)+U_{2}(y)$ and judge the existence of an incentive compatible, individual rational, and ex-post efficient mechanism by the sign of the minimum value.

This time, we cannot prove the non-existence theorem in reference [1] and [9], because we can easily construct a special case to prove its existence. Although the area doesn't satisfy the convex and differentiable assumption, the example is indeed the simplest way to construct such a special case. Since the convex and differentiable assumptions are not substantially essential to our main result, we can release these assumptions temporary.

In the case shown in Figure 3, the whole area $D$ consists of two rectangles: $D$ and $S$. $D:\left[a_{1}, b_{1}\right] \times\left[a_{2}, b_{2}\right], S:\left[a_{1}, c_{1}\right] \times\left[a_{2}, c_{2}\right]$ and $c_{2}<b_{1}$.

On $D$, the density function has form:

$$
\left.f\left(v_{1}, v_{2}\right)\right|_{D}=f_{1}\left(v_{1}\right) \times f_{2}\left(v_{2}\right)
$$

Also, denote the corresponding "distribution" functions (not strict distribution functions because they don't satisfy: $\left.F_{i}(\infty)=1\right)$ as $F_{1}\left(v_{1}\right), F_{2}\left(v_{2}\right)$ as the integral function of $f_{1}\left(v_{1}\right)$ and $f_{2}\left(v_{2}\right)$, which implies $V_{1}$ and $V_{2}$ are "locally" independent on $D$.

We need some new notations to state the special case in order.

$$
\begin{gathered}
r=\iint_{D} f\left(v_{1}, v_{2}\right) \mathrm{d} v_{1} \mathrm{~d} v_{2} \\
g\left(v_{1}, v_{2}\right)=\left.f\left(v_{1}, v_{2}\right)\right|_{S} \\
G_{2}\left(v_{2}\right)=\int_{a_{2}}^{v_{2}} g_{2}(x) \mathrm{d} x, G_{1}\left(v_{1}\right)=\int_{a_{1}}^{v_{1}} g_{1}(x) \mathrm{d} x \\
g_{1}\left(v_{1}\right)=\int_{a_{2}}^{c_{2}} g\left(v_{1}, t\right) \mathrm{d} t, g_{2}\left(v_{2}\right)=\int_{a_{1}}^{c_{1}} g\left(v_{2}, t\right) \mathrm{d} t
\end{gathered}
$$

If we assume $c_{1}$ is so small such that the minimum value of $\left(U_{1}(x)+U_{2}(y)\right)$ achieves at $U_{1}\left(b_{1}\right)+U_{2}\left(c_{2}\right)$.

Then we can obtain the minimum utility:

$$
\begin{aligned}
& \min \left(U_{1}(x)+U_{2}(y)\right)=U_{1}\left(b_{1}\right)+U_{2}\left(c_{2}\right) \\
& =-r \int_{c_{2}}^{b_{1}}\left(1-F_{2}(t)\right) F_{1}(t) \mathrm{d} t+\iint_{S} \frac{G_{2}\left(v_{2}\right)}{g_{2}\left(v_{2}\right)}-\frac{G_{1}\left(v_{1}\right)}{g_{1}\left(v_{1}\right)} p\left(v_{1}, v_{2}\right) g\left(v_{1}, v_{2}\right) \mathrm{d} v_{1} \mathrm{~d} v_{2}
\end{aligned}
$$

Minimum utility can be positive if $r$ is very small and $\frac{G_{2}\left(v_{2}\right)}{g_{2}\left(v_{2}\right)}-\frac{G_{1}\left(v_{1}\right)}{g_{1}\left(v_{1}\right)}$ is 


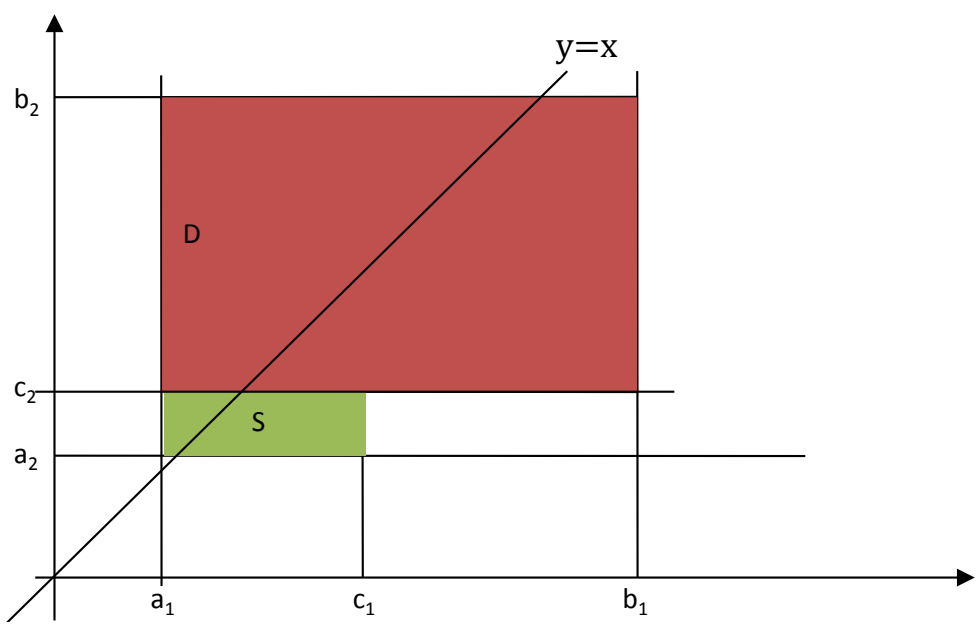

Figure 3. The simplified case where an IC IR EPE mechanism may exist.

large enough. These conditions can be satisfied if we intend to construct such a density function.

We have proved that under generalized bilateral trading model, there may exist an incentive-compatible individual rational and ex-post efficient mechanism. Anyway, the economic intuition about the generalized model should also be declared. Still, some empirical and further theoretical analyses are also needed to be made.

\section{Further Interpretations}

After the proof of the possible existence of an incentive-compatible individual rational and ex-post efficient mechanism in the generalized bilateral trading model, now we turn our attention to the empirical explanation to the key assumption, which is dealing with the distribution of the agents' type.

Empirically, since beliefs of people vary from person to person, different types of individual also face different choice of their seller in the case of bilateral trading. That is a possible explanation for our modified assumptions. A typical example is: if you want to buy a car, you can buy it at second-hand car market but you can also buy it at flagship store. It's an often case that wealthy consumers buy their cars at flagship store while the poor not. This phenomenon results in that different cohorts are matched if consumers don't share the same type (willingness-to-pay). Especially, When assuming there are a large number of such markets. The trading area will be restricted to the generalized trading model, where types are not independent by the updated belief or rational expectation.

There is another question: if we can buy the same thing at various market. It will be a dominant strategy to choose a market that has relative low expected valuation of the object. Under this assumption, the high-end market will crash and all buyers will purchase goods at low-end market or second-hand market, while this is obviously not the fact.

The generalized market may have an incentive compatible, individual rational 
and ex post efficient mechanism theoretically, but we can further analysis the empirical data to decide whether there exists an incentive compatible, individual rational and ex post efficient mechanism for each market.

Empirically, we can study the bilateral trading results in various markets to provide supporting evidences for the generalized model. We should first specify how many kinds of markets exist in all and examine the trading data of each market to obtain an unbiased estimation of area $D$. Then we find a best differentiable area $D^{\prime}$ that can be treated as an approximation of $D$.

Then we need to estimate the corresponding density function $f\left(v_{1}, v_{2}\right)$ by sampling and calculate the minimum value of $\left(U_{1}(x)+U_{2}(y)\right)$ by finding the point satisfying first order condition on the boundary of $D$ '. Using the above result, we can identify whether an incentive compatible, individual rational, ex post efficient mechanism can exist.

Theoretically, finding the sufficient and necessary conditions of the existence of an incentive compatible, individual rational, and ex post efficient mechanism is still an unfinished work.

\section{Conclusion}

In conclusion, this paper successfully generalizes the bilateral trading model by easing the restriction on the distribution, which now can be non-independent. The two main changes brought by the modification are: 1) the expression of the expected utility with respect to each player; 2 ) the changes in determining condition for individual rationality. These two changes make it possible for the existence of an incentive compatible, individual rational, and ex post efficient mechanism.

\section{References}

[1] Myerson, R.B. and Satterthwaite, M.A. (1981) Efficient Mechanisms for Bilateral Trading. Journal of Economic Theory, 29, 265-281. https://doi.org/10.1016/0022-0531(83)90048-0

[2] Spence, M. (1973) Job Market Signaling. Quarterly Journal of Economics, 87, 355-374. https://doi.org/10.2307/1882010

[3] Akerlof, G.A. (1970) The Market for Lemons: Quality Uncertainty and the Market Mechanism. The Quarterly Journal of Economics, 84, 488-500. https://doi.org/10.2307/1879431

[4] Rothschild, M. and Joseph, S. (1976) Equilibrium in Competitive Insurance Markets: An Essay on the Economics of Imperfect Information. The Quarterly Journal of Economics, 90, 629-649. https://doi.org/10.2307/1885326

[5] Myerson, R.B. (1981) Optimal Auction Design. Mathematics of Operations Research, 6, 58-73. https://doi.org/10.1287/moor.6.1.58

[6] Vickrey, W. (1961) Counter Speculation, Auctions, and Competitive Sealed Tenders. Journal of Finance, 16, 8-37. https://doi.org/10.1111/j.1540-6261.1961.tb02789.x

[7] Chatterjee, K. (1982) Incentive Compatibility in Bargaining under Uncertainty. The Quarterly Journal of Economics, 97, 717-726. https://doi.org/10.2307/1885109 
[8] Riordan, M.H. (1984) Uncertainty, Asymmetric Information and Bilateral Contracts. The Review of Economic Studies, 51, 83-93. https://doi.org/10.2307/2297706

[9] Myerson, R.B. (1979) Incentive Compatibility and the Bargaining Problem. Econometrica, 47, 61-73. https://doi.org/10.2307/1912346 